\title{
Eine syntaktische Analyse von Sprechen und Blickverhalten bei dyadischer Interaktion
}

\author{
A. H. Clarke, H. Wagner, J. H. Ellgring, München
}

\section{Einführung:}

Strukturelle Ansätze wurden für die Untersuchung sozialer Interaktion im Bereich der Soziologie und der Psychologie vielfach verwendet. Die verschiedenen deskriptiven Arbeiten von Goffman (1955) über die Natur und Funktion der sozialen Rituale und von Miller et al. (1960) über die Struktur und die „Grammatik“ des Verhaltens sind klassische Beispiele dafür.

Grundlegend für diese und andere Arbeiten ist das Konzept des regelgeleiteten Verhaltens. Vor allem in den verschiedenen Bereichen der Sprachwissenschaft - von der Sprechakttheorie (Searle, 1969) bis zur syntaktischen Mustererkennung von Silben (Fu, 1977) - ist dieser Ansatz geläufig. Auf ihre fundamentale Bedeutung für die strukturelle Linguistik braucht man hier nicht weiter einzugehen.

Die Anwendung eines strukturellen Ansatzes auf nichtverbales kommunikatives Verhalten ist in der Literatur wenig verfolgt worden. Die Arbeiten von Birdwhistell (1970), Duncan (1973) und Nowakowska (1976) weisen zwar auf die Relevanz der linguistischen oder grammatischen Analogie hin, bleiben aber ohne breite Stützung durch empirische Untersuchungen. Argyle/ Kendon (1967) diskutieren einige experimentelle Studien sozialer Interaktion, die einen strukturellen Ansatz zur Beschreibung des kommunikativen Verhaltens stützen.

Akzeptiert man die aus der Ethologie entwickelten Argumente, die für die hierarchische Natur der Verhaltensorganisation sprechen - wie sie z. B. von Pruscha/Maurus (1976) oder Dawkins (1976) dargestellt wurden - dann scheint es plausibel, daß dieses Konzept regelgeleiteten Verhaltens im Verlauf sozialer Interaktion in gewissem Maße auf alle relevanten Ebenen verbalen und nichtverbalen Verhaltens anwendbar ist.

Die Verwendung solcher Konzepte für die Analyse nichtverbaler Verhaltenskomponenten in der dyadischen Situation soll hier besprochen werden. Im besonderen werden die Beziehungen zwischen Sprech- und Blickaktivität untersucht. Die Bedeutung der Koordination von Sprech- und Blickaktivität beim Interaktionsprozeß ist durch viele Arbeiten offenkundig geworden (siehe Argyle/Cook 1974). Die Mehrzahl solcher Arbeiten konzentriert sich auf die statistische Beschreibung oder auf den Gebrauch rein stochastischer Modelle. Ein derartiger Ansatz liefert zwar wesentliche Informationen über den Prozeß, aber keine adäquate Beschreibung der möglichen regelgeleiteten Beziehungen zwischen Elementen wie sie etwa ein grammatisches Modell liefert. Andererseits beruhen Arbeiten, in denen auf solche strukturellen Beziehungen eingegangen wird (Duncan 1973), auf einer schmalen Datenbasis. 
Wir gehen von der Hypothese aus, daß eine Untersuchung der zeitlichen Koordination von Sprech- und Blickaktivität Ergebnisse liefern sollte, die mit einem strukturellen Ansatz in Einklang zu bringen sind. An einen derartigen Ansatz, wie er in der Mustererkennungsliteratur verstanden wird, ist die Erwartung geknüpft, daß das strukturelle Modell Aufschluß über den zugrundeliegenden oder generierenden Mechanismus gibt.

Zusätzlich wird hier der Standpunkt vertreten, daß weder ein rein stochastisches noch ein rein strukturelles Modell zufriedenstellend für die praktische Anwendung sein kann. Vielmehr muß ein Konzept entwickelt werden, das sowohl die Regelmäßigkeit des Verhaltens als auch die Variation in den beobachteten Sequenzen von Verhaltenseinheiten berücksichtigt. In diesem Sinne würden die Regeln, die den Ablauf einer spezifischen sozialen Interaktion bestimmen, durch verschiedene Faktoren mehr oder weniger beeinflußt - z. B. die Befindlichkeit und das Rollenverständnis der Interaktionspartner oder die soziale Umgebung.

Nach dieser Vorstellung werden die Verhaltenssequenzen auf einer regelgeleiteten oder grammatischen Ebene generiert und zum gewissen Grad durch die genannten Faktoren ,,verrauscht". Es wird später auf dieses Konzept eingegangen.

\section{Beobachtungsmaterial}

Der Großteil der Daten für die hier besprochene Analyse wurde durch Beobachtung von klinisch-psychiatrischen Interviews gewonnen, die auf Videoband gespeichert wurden. Die Interviews wurden im Rahmen einer Längsschnittuntersuchung depressiver Patienten aufgezeichnet und werden auf verschiedene Weise analysiert zur Feststellung von Anderungen des Sozialverhaltens im Verlauf einer depressiven Erkrankung (Ellgring \& Clarke, 1978). Für jeden Patienten wurde eine Serie von Aufzeichnungen gemacht, die durchschnittlich zwanzig Interviews umfaßt. Diese Interviews wurden in regelmäßigen Zeitabständen während des stationären Aufenthalts in der Klinik durchgeführt.

Weitere Daten wurden aus Gesprächen von Partnern gewonnen, die sich einer Partnertherapie unterziehen wollen.

Die Datensammlung entspricht in etwa der Art, wie sie Kendon (1967) und Argyle (1969) mit einem Interaction Chronograph vorgenommen haben (Abb. 1). Es wurden jedoch beträchtliche Verbesserungen hinsichtlich der Genauigkeit und der Zeitersparnis erreicht, indem ein synchronisierter Video-Zeitcode bei der Aufnahme mitaufgezeichnet wurde. Dadurch wurde eine Registrierung der Beobachtungsdaten zusammen mit den dazugehörigen Zeitcode-Informationen im Computer möglich. Auf diese Weise wird jede beobachtete Interviewsituation (ca. 5 min. lang) durch eine Sequenz von diskreten Verhaltenszuständen repräsentiert, wobei ein Repertoire von insgesamt 16 Zuständen möglich wäre. Die Zustände ergeben sich aus den sechzehn möglichen Kombinationen der vier gezeigten Binärkanäle (s. Abb. 1). 




Abb. 1: Schema der Kodierung. Dieser Ausschnitt eines Beobachtungsprotokolls zeigt wie die verschiedenen Kanäle (Sprechen A,B; Blicken A,B) von Beobachtern kontinuierlich als on-off patterns registriert werden. Neben der direkten Darstellung der Kanäle sind die Binär- und Dezimalkodierungen der Zustände angegeben. Das Erfassungsprogramm speichert diese Zustandskodierungen und die Zeitpunkte ihres Auftretens. Auf diese Weise werden alle Informationen über die vier Kanäle festgehalten, die für die nachfolgende Analyse notwendig sind. Die pik tographischen Darstellungen dienen zur Veranschaulichung der registrierten Zustände: links jeweils Partner A, rechts jeweils Partner B. Beim ersten Zustand spricht Partner A und blickt Partner B an. Partner B schweigt und blickt weg.

\section{Graphische Darstellung}

Für die Darstellung der Ergebnisse betrachten wir die relative Dauer jedes Zustandes - soweit er vorkommt - und die Ubergangshäufigkeiten zwischen diesen Zuständen. Die sechzehn möglichen Zustände des Verhaltensrepertoires können in vier Gruppen geordnet werden, die den vier möglichen Sprechbedingungen entsprechen, nämlich: (a) gemeinsames Schweigen, (b) Sprechen von Person A, (c) Sprechen von Person B, (d) gemeinsames Sprechen.

Jede dieser vier Sprechbedingungen kann wiederum in die vier möglichen Blickbedingungen unterteilt werden, nämlich: (a) keiner blickt den anderen an, (b) Person A blickt B an, (c) Person B blickt A an, (d) wechselseitiges An- 
blicken. Um diese Anordnung verständlicher zu machen, ist sie in dem Piktogramm in Abb. 2 graphisch dargestellt.
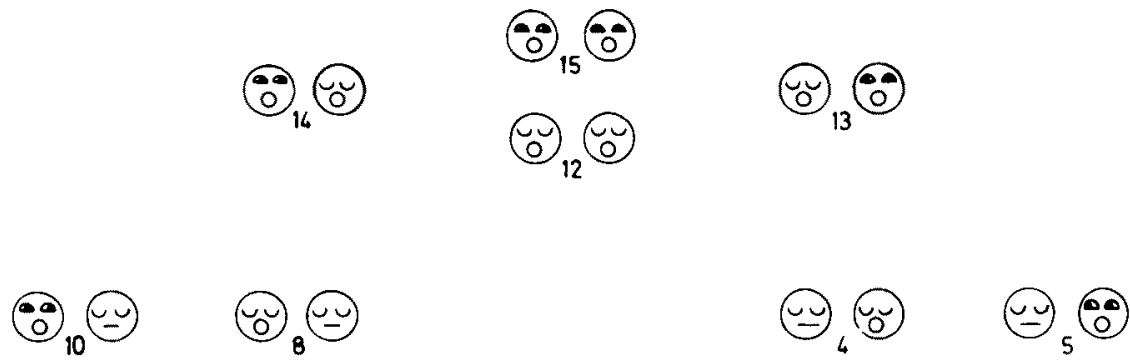

(a)
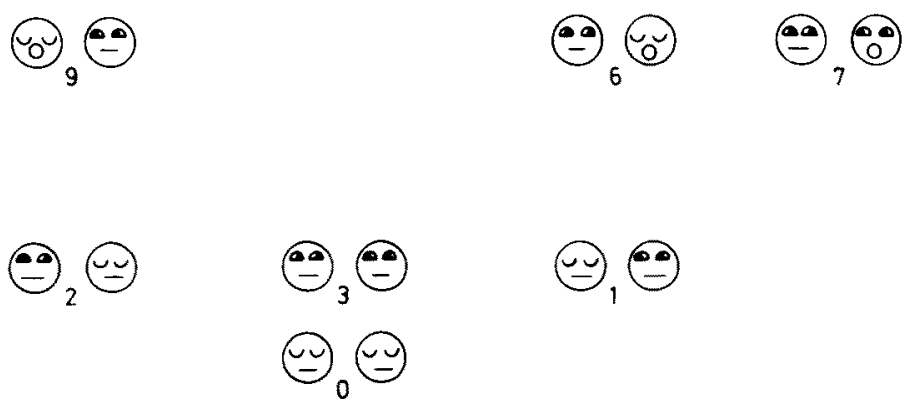

Abb. 2: Piktogramm der 16 theoretisch möglichen Zustände: Die Zustände sind nach dem Sprechverhalten der Partner in Vierergruppen gegliedert.

Zustand $0-3$ : Beide schweigen

Zustand 4- 7: Partner B spricht

Zustand 8-11: Partner A spricht

Zustand 12-15: Gleichzeitiges Sprechen

In jeder Gruppe sind die vier möglichen Blickzustände der Dyade enthalten.

Entsprechend der Anordnung des Piktogramms werden für jere beobachtete Situation die gemessenen Verhaltensweisen folgendermaßen dargestellt: die relative Zeitdauer jedes vorkommenden Zustandes wird durch einen Kreis dargestellt, dessen Flächengröße nach dem relativen Verweildauer proportioniert ist. Die Kreise werden nach dem Piktogrammschema positioniert. Die Ubergangshäufigkeiten werden durch Pfeile repräsentiert, deren Stärke nach der relativen Ubergangshäufigkeit proportioniert ist. Beispiele von zwei solchen ausgewerteten Situationen zeigen die Abbildungen 3 und 4. 

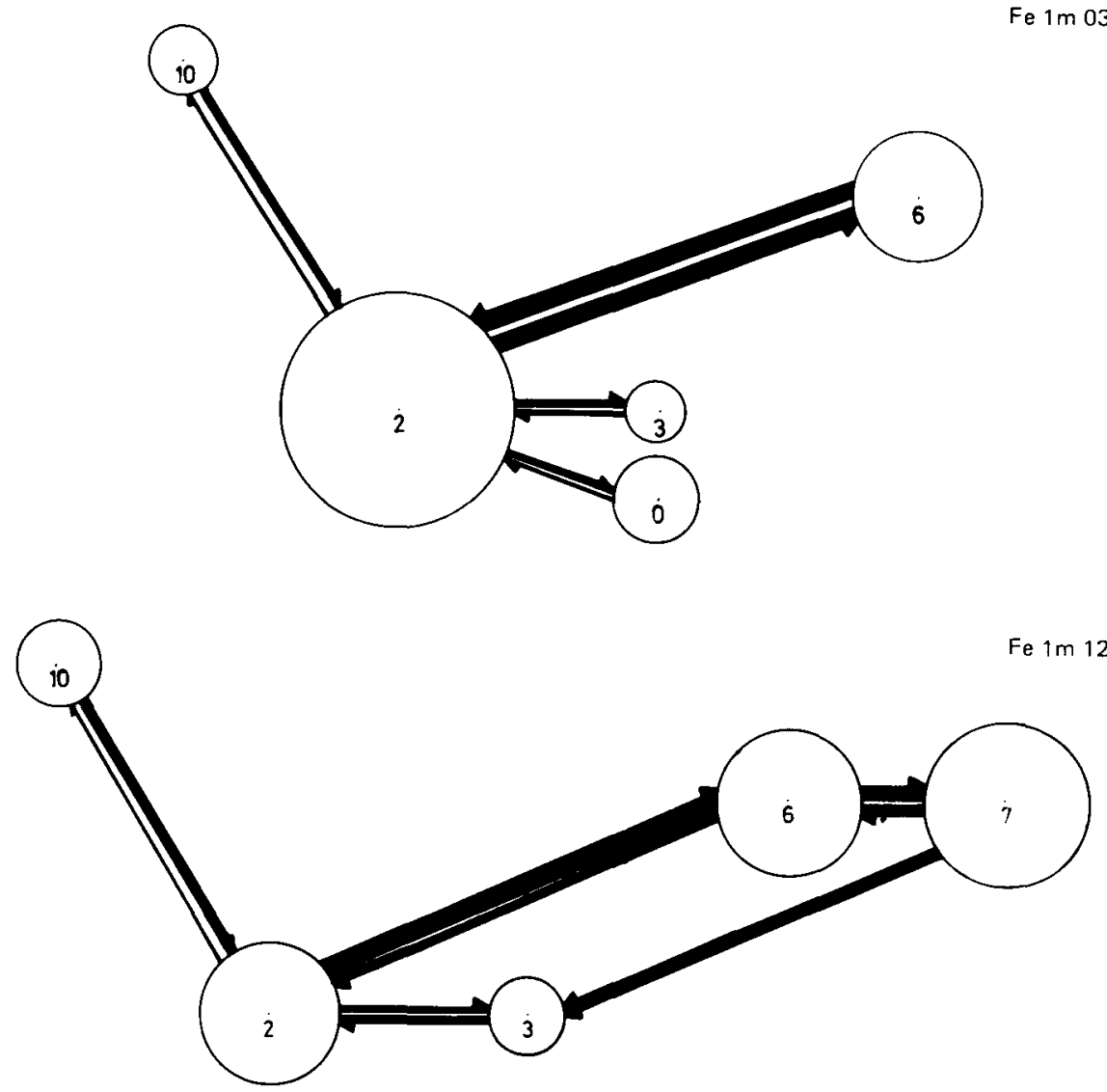

Abb. 3: Klinische Interviewsituationen: Graphische Darstellung der Verweildauer in und Übergangshäufigkeiten zwischen den vorkommenden Zuständen. (Die Anordnung entspricht der Abbildung 2).

Der Flächengröße der Kreise entspricht die gesamte Verweildauer im jeweiligen Zustand; die Stärke jedes Pfeils entspricht der Übergangshäufigkeit zwischen den jeweiligen Zuständen.

a) entstammt einem Interview aus der Zeit schwerer Depression.

b) entstammt einem Interview kurz vor der Entlassung.

Abb. 3 entstammt einem klinischen Interview aus der Zeit, in der sich der Patient im Zustand schwerer Depression befand. Abb. 4 stellt einen Ausschnitt aus einem Gespräch zwischen einem Paar dar, das vor Beginn einer Partnertherapie aufgezeichnet wurde.

In dem Fall des Partnergespräches ist die „Floortime“ annähernd gleich auf die Gesprächspartner verteilt. (,,Floortime“ oder „Turn“ wird hier verstanden als die Zeit, die ein Teilnehmer für sich beansprucht oder die ihm freigestellt wird.) Der Wechsel der Sprecher ist schnell; wenig oder keine Zeit wird schweigend verbracht, und die Sprecherwechsel erfolgen meist 
über die komplementären Zustände - (13 und 14) - die gleichzeitiges Sprechen beinhalten. Das mögliche Repertoire von Verhaltenszuständen wird zum großen Teil genutzt ( 11 und 16 Zuständen werden besetzt).

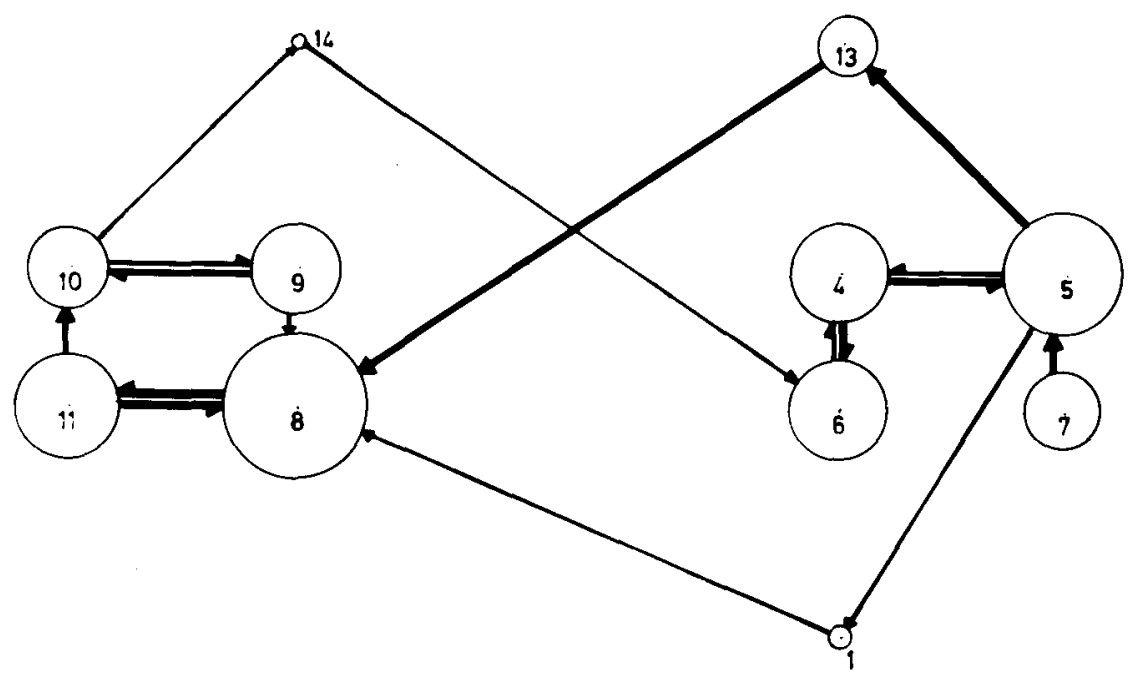

Abb. 4: Partnergespräch: Graphische Darstellung der Verweildauer in und Ubergangshäufigkeiten zwischen den vorkommenden Zuständen (siehe Abb. 3 für eine ausführlichere Erklärung).

Das Interviewbeispiel dagegen weist eine stark ungleiche Verteilung der „Floortime" auf, wobei der Interviewer einen wesentlich geringeren Anteil beansprucht. Der Wechsel der Sprecher erfolgt über Zustand 2 (Schweigen), und ein kleinerer Teil des möglichen Repertoires wird ausgenutzt (5 von 16 möglichen Zuständen).

\section{Syntaktischer Ansatz}

Wendet man das Konzept regelgeleiteten Verhaltens auf eine dyadische Interaktion an, so kann man davon ausgehen, daß während einer solchen Interaktion jeder Teilnehmer eine bestimmte Rolle übernimmt und sich gemäß seinem Rollenverständnis regelgeleitet verhält. Daher sollten die Konsequenzen dieser Regelbefolgung in verbalen und nichtverbalen Verhaltensweisen beobachtbar sein. Es ist anzunehmen, daß die Teilnehmer auch diese Verhaltensweisen als Bestandteil ihrer Rolle betrachten. Auf der Verhaltensebene, die hier für die Datengewinnung beobachtet wurde, muß außer diesem Rollenverständnis mindestens ein anderer gewichtiger Faktor in Betracht gezogen werden: die kognitive Belastung des Individuums (im Sinne der Auf- 
merksamkeitsstruktur von Chance, 1962). Genauer gesagt, die gleichzeitige Belastung der kognitiven Kapazität durch die Verarbeitung der sichtbaren Signale aus der sozialen Umgebung sonstiger Information. Dazu gibt es eine Reihe von Berichten, u. a. von Kendon (1967).

Das beobachtete Verhalten, nämlich Sprech- und Blickaktivität, ist somit sowohl vom Rollenverständnis der Teilnehmer als auch von ihrer kognitiven Belastung bestimmt. Unter dieser Voraussetzung sollte die Struktur der Beobachtungsdaten nicht nur diejenigen Regeln abbilden, die von den Teilnehmern befolgt wurden, sondern auch das Ausmaß angeben, in dem die Teilnehmer durch die Situation und durch ihre eigene innere Verfassung kognitiv belastet werden.

Gemäß diesen Überlegungen wurden einige Konzepte der Linguistik auf die Untersuchung der besprochenen Verhaltensebene angewendet. Zum einen werden die möglichen Verhaltenszustände, $\mathrm{d}$. h. die 16 möglichen Kombinationen der vier beobachteten Verhaltenskanäle als die elementaren syntaktischen Einheiten betrachtet. Zusammengenommen repräsentieren sie das Repertoire des Verhaltens. Zweitens wird davon ausgegangen, daß die beobachteten Ketten solcher Einheiten nach den Ersetzungsregeln einer Grammatik bestimmt werden. Drittens wird jeder Regel eine bestimmte Wahrscheinlichkeit zugeordnet, weil wir uns auch für die unterschiedlichen Häufigkeiten interessieren, mit denen die beobachtete Kette - und daher auch die einzelnen Regeln - in verschiedenen Situationen vorkommen.

Ein weiterer wichtiger Punkt ist die Definition der Analyseeinheit. Während dies für die Sprache im allgemeinen der Satz ist, wird für die nichtverbale Interaktion generell ein Austausch zwischen den Teilnehmern als Analyseeinheit genommen. Für jede der schon besprochenen Situationen wurde auf diese Weise eine probabilistische Grammatik aufgestellt (s. Anhang). Diese Beispiele zeigen deutlich die Wichtigkeit der drei genannten Merkmale eines derartigen Modells, nämlich das Repertoire bzw. das terminale Vokabular $\left(V_{T}\right)$, die Ersetzungsregeln und deren Wahrscheinlichkeiten.

Mit den Grammatiken konnten die Unterschiede, die aus der graphischen Darstellung interpretierbar sind, quantitativ dargelegt werden.

Um die strukturelle Beschreibung bzw. die Ersetzungsregeln festzulegen, wurde zuerst die hierarchische Aufteilung, die ähnlich der graphischen Anordnung (Abb. 2) differenziert wird, herangezogen. Auf diese Weise kann der Dialog (D) hinsichtlich der Zeitintervalle, die der ,Floortime“ oder der „Turns" von Person A (nichtterminales Element: A) bzw. von Person B (nichtterminales Element: B) entsprechend aufgeteilt werden. Die Definition oder genaue Abgrenzung dieser Intervalle bereitet auf dieser Ebene allerdings Schwierigkeiten. Man ist gezwungen, eine weitere Kategorie einzuführen, die den Wechsel der Sprecher repräsentiert (nichtterminales Element: Ch). Die „Floortime“ oder „Turn“ Kategorie kann für jeden Teilnehmer weiter aufgeteilt werden, so daß eine Differenzierung in Zustände, in denen die Person tatsächlich spricht (As, Bs), und in Zustände, in denen gar nicht gesprochen wird $(\mathrm{Pa})$, eingeführt wird. Die Bestimmung des terminalen Vokabulars und die Zuordnung der terminalen Zeichen zu den erwähnten nichtterminalen Kategorien erfolgen durch empirische Untersuchung der spezifischen Situationen. 




Abb. 5: Syntaktische Strukturbeschreibung für eine einfache dreier Kette in Form eines Stammbaumes.

Für den Fall, daß die Ersetzungsregeln der Grammatik bekannt sind, reduziert sich das Problem darauf, eine optimale Schätzung für die Wahrscheinlichkeiten zu liefern. Von den beobachteten Sequenzen werden zunächst die Häufigkeiten der Ketten von 3, 4, 5 usw. aufeinanderfolgenden Zuständen ausgezählt, die in der betrachteten Stichprobe vorkommen. Für jede beobachtete Kette wird unter Verwendung der vereinbarten Ersetzungsregeln ein Stammbaum aufgestellt, der der strukturellen Beschreibung entspricht (s. Abb. 5). Dadurch können für die Stichprobe die Häufigkeiten der Anwendung jeder Ersetzungsregel festgestellt und die entsprechenden Wahrschein. lichkeiten ausgerechnet werden. Die Berechnung dieser Wahrscheinlichkeiten erfolgt durch eine Maximum-Likelihood Schätzung. Die berechneten Grammatiken sind in den Abbildungen 6 und 7 aufgestellt. 


\section{Interview: $\mathrm{Fe} 1 \mathrm{mo3}$}

$G=\left(V_{N}, V_{T}, P, S, p\right)$

$V_{N}=\left(D, A, B, C h, P a, A_{5}, B_{S}\right)$

$V_{T}=(0,2,3,6,10)$

$s=v$

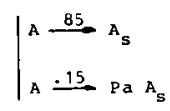

$\mid \begin{aligned} & \mathrm{B} \stackrel{.46}{\longrightarrow} \\ & \mathrm{B} \\ & \stackrel{.54}{\longrightarrow}\end{aligned}$

$A_{s} \stackrel{1.0}{=} 10$

$B_{s} \stackrel{1 . Q}{=} 6$

$\left\{\begin{array}{l}\mathrm{Pa} \stackrel{.22}{\longrightarrow} 2 \mathrm{~B} \\ \mathrm{~Pa} .62-2 \\ \mathrm{~Pa} \stackrel{.07}{\longrightarrow} 23 \\ \mathrm{~Pa} .09-20\end{array}\right.$

| ch $\stackrel{1.0}{-} 2$

\section{Interview: $\mathrm{Fe} 1 \mathrm{~m} 12$}

$G=\left(v_{N}, V_{T}, P, S, P\right)$

$v_{\mathrm{N}}=\left(\mathrm{D} ? \mathrm{~A}, \mathrm{~B}, \mathrm{Ch}, \mathrm{Pa}, \mathrm{A}_{\mathbf{s}}, \mathrm{B}_{\mathbf{s}}\right)$

$v_{T}=(2,3,6,7,10)$

$\mathrm{S}=\mathrm{D}$

$\mid \begin{aligned} & \mathrm{A} \stackrel{.86}{\longrightarrow} \mathrm{A}_{\mathrm{S}} \\ & \mathrm{A} \stackrel{.14}{\longrightarrow} \mathrm{Pa} \mathrm{A}_{\mathrm{S}} \quad \mathrm{A}_{\mathrm{S}} \stackrel{1.0}{\longrightarrow} 10\end{aligned}$

$\mid \begin{array}{ll}\mathrm{B} \stackrel{.40}{\longrightarrow} \mathrm{B}_{\mathrm{S}} & \\ \mathrm{B} \stackrel{.53}{\longrightarrow} \mathrm{B}_{\mathrm{S}} \mathrm{Pa} & \mathrm{B}_{\mathrm{S}} \stackrel{.60}{\longrightarrow} 6 \\ \mathrm{~B} \stackrel{.07}{\longrightarrow} \mathrm{B}_{\mathrm{s}}^{\mathrm{B}} & \mathrm{B}_{\mathrm{s}} \stackrel{.40}{\longrightarrow} 7\end{array}$

$\mid \begin{aligned} & \mathrm{Pa} \stackrel{.54}{2} 2 \\ & \mathrm{~Pa} \stackrel{.46}{\longrightarrow} 3\end{aligned}$

$\mid \begin{aligned} & \mathrm{Ch} \stackrel{.89}{\longrightarrow} 2 \\ & \mathrm{Ch}-11\end{aligned}$

Abb. 6a,b: Formale Aufstellung der berechneten probabilistischen Grammatiken für die zwei besprochenen Interviews.

$$
\begin{aligned}
& \text { Gesprach: } 5455 \\
& G=\left(v_{\mathrm{N}}, v_{T}, P, S, p\right) \\
& V_{N}=\left(D, A, B, C h, A_{S}, B_{S}\right) \\
& v_{T}=(1,4,5,6,7,8,9,10,11,13,14) \\
& S=D \\
& \left.\right|_{A \stackrel{.44}{\longrightarrow} A_{S}} ^{A .56} A_{S} A \\
& \mid \begin{array}{l}
\mathrm{B} \stackrel{.49}{\longrightarrow} \mathrm{B}_{\mathrm{S}} \stackrel{.51}{\longrightarrow} \mathrm{B}_{\mathrm{S}} \mathrm{B}
\end{array} \\
& \mid \begin{array}{l}
\mathrm{A}_{\mathrm{s}} \stackrel{.12}{\longrightarrow} 8 \\
\mathrm{~A}_{\mathrm{s}} \stackrel{.44}{\longrightarrow} 9 \\
\mathrm{~A}_{\mathrm{s}} \stackrel{.20}{\longrightarrow} 10 \\
\mathrm{~A}_{\mathrm{s}} \stackrel{.24}{\longrightarrow} 11
\end{array} \\
& \mid \begin{array}{l}
\mathrm{B}_{\mathrm{s}} \stackrel{.29}{\longrightarrow} 4 \\
\mathrm{~B}_{\mathrm{s}} \stackrel{.19}{\longrightarrow} 6 \\
\mathrm{~B}_{\mathrm{s}} \stackrel{.40}{\longrightarrow} 5 \\
\mathrm{~B}_{\mathrm{s}} \stackrel{.12}{\longrightarrow} 7
\end{array} \\
& \mathrm{Ch} \stackrel{.41}{\longrightarrow} 13 \\
& \mathrm{Ch} \stackrel{.20}{-1} \\
& \mathrm{Ch} \stackrel{39}{\longrightarrow}-14
\end{aligned}
$$

Abb. 7: Formale Aufstellung der probabilistischen Grammatik für das Partnergespräch. 
Mit dieser Arbeit wurde die Frage angegangen, ob die Organisation der nichtverbalen Interaktion analog zu einer linguistischen Struktur behandelt werden kann.

Die entwickelte graphische Darstellung zeigt eine prägnante Gestalt der Beziehungen zwischen den beobachteten Verhaltenszuständen. In ihr bilden sich sowohl situative Unterschiede als auch Veränderungen im internen Zustand der Teilnehmer ab. Die Darstellung beruht auf der Verweildauer der einzelnen vorkommenden Zustände und den Übergangshäufigkeiten erster Ordnung. Darüber hinaus deutet sie auf eine Struktur höherer Ordnung hin. Sie bildet somit den Ausgangspunkt für die formale Strukturbeschreibung der Verhaltenssequenzen in Form einer Grammatik. Konkret wurde das Modell einer probabilistischen Grammatik verwendet. Im Rahmen eines derartigen Modells wird das beobachtete Verhaltensrepertoire durch eine Menge von Ersetzungsregeln strukturell beschrieben. Den Ersetzungsregeln werden empirisch bestimmte Wahrscheinlichkeiten zugeordnet, um die ,verrauschte“ Natur der Daten zu berücksichtigen. Diese grammatischen Modelle erfassen situative Unterschiede und Veränderungen des internen Zustands der Teilnehmer differenzierter und sind geeigneter für eine weiterführende quantitative Auswertung als graphische Darstellungen. Insgesamt macht dieser Ansatz die Regelhaftigkeit der nichtverbalen Interaktion evident.

\section{Anhang:}

Eine probabilistische Grammatik wird definiert als ein Quintuple:

$$
\mathrm{G}=\left(\mathrm{V}_{\mathrm{N}}, \mathrm{V}_{\mathrm{T}}, \mathrm{P}, \mathrm{S} . \mathrm{p}\right) \text {, }
$$

wobei $V_{N}$ eine endliche Menge von nichtterminalen Zeichen,

$V_{\mathrm{T}}$ eine endliche Menge von terminalen Zeichen,

$\mathrm{P}$ die Menge von Produktionen bzw. Ersetzungsregeln,

S das Startsymbol,

p das Wahrscheinlichkeitsmaß über die Menge von Produktionen ist.

Auf diese Weise besteht jede Produktion aus drei Elementen $\left(\alpha_{\mathrm{i}}, \beta_{\mathrm{i}}, \mathrm{p}_{\mathrm{ij}}\right)$ wo $p_{\mathrm{ij}}$ eine reale $\mathrm{Zahl}$ ist, die die Wahrscheinlichkeit angibt, daß $\alpha_{\mathrm{i}}$ durch $\beta_{\mathrm{j}}$ ersetzt wird.

Während eine normale Grammatik eine strukturelle Beschreibung der möglichen Ketten liefert, gibt eine probabilistische Grammatik darüber hinaus eine Wahrscheinlichkeit für jede Ersetzungsregel an.

\section{Literatur}

Argyle, M./Cook, M., 1976: Gaze and Mutual Gaze. London.

Argyle, M./Kendon, A., 1967: The experimental analysis of social performance, in: L.

Berkowitz, ed., 1967: Advances in Experimental Social Psychology, New York/London, S. 55-98.

Birdwhistell, R., 1970: Kinesics and Context, Philadelphia. 
Chance, M. R. A., 1962: The interpretation of some agonistic postures, in: Evolutionary aspects of animal communication, 8, S. 71-89.

Dawkins, R., 1976: Hierarchical Organisation: a candidate principle for ethology. In: P. P. G. Bateson/R. A. Hinde, eds, 1976: Growing Points in Ethology, London.

Duncan, S., 1973: Toward a grammar for dyadic conversations, in: Semiotica 9, S. 2946.

Ellgring, J. H., 1976: Blickverhalten und Sprechaktivität. München.

Ellgring, J. H./Clarke, A. H., 1978: Verlaufsbeobachtungen anhand standardisierter Videoaufzeichnungen bei depressiven Patienten. In: Helmchen, H./Renfordt, E., Hsg., 1978: Fernsehen in der Psychiatrie, Stuttgart.

Fu, K. S., 1977: Introduction to Syntactic Pattern Recognition. In: K. S. Fu, ed., 1977: Syntactic Pattern Recognition.

Goffman, E., 1955: On Facework, in: Psychiatry 18, S. 213-231.

Kendon, A, 1967: Some functions of gaze direction in social interaction, in: Acta Psychologica 26, S. 22-63.

Pruscha, H.Maurus, M., 1976: The communicative function of some agonistic behaviour patterns in squirrel monkeys: The relevance of social context, in: Behav. Ecol. Sociobiol. 1, S. $185-214$.

Miller, G. A./Galanter, E. A./Pribram, K. H., 1960: Plans and the Structure of Behavior. New York.

Nowakowska, M., 1976: Towards a formal theory of dialogues, in: Semiotica 7, S. 291 313.

Searle, J., 1969: Speech Acts. London. 